\title{
Electrochemical Aptasensor Based on Prussian Blue-Chitosan-Glutaraldehyde for the Sensitive Determination of Tetracycline
}

\author{
Guanghui Shen, Yemin Guo, Xia Sun*, Xiangyou Wang
}

(Received 01 November 2013; accepted 07 January 2014; published online 20 March 2014)

\begin{abstract}
In this paper, a novel and sensitive electrochemical aptasensor for detecting tetracycline (TET) with prussian blue $(\mathrm{PB})$ as the label-free signal was fabricated. A PB-chitosan-glutaraldehyde (PB-CS-GA) system acting as the signal indicator was developed to improve the sensitivity of the electrochemical aptasensor. Firstly, the PB-CS-GA was fixed onto the glass carbon electrode surface. Then, colloidal gold nanoparticles (AuNPs) were droped onto the electrode to immobilize the anti-TET aptamer for preparation of the aptasensor. The stepwise assembly process of the aptasensor was characterized by cyclic voltammetry (C-V) and scanning electron microscope (SEM). The target TET captured onto the electrode induced the current response of the electrode due to the non-conducting biomoleculars. Under the optimum operating conditions, the response of differential pulse voltammetry (DPV) was used for detecting the concentration of TET. The proposed aptasensor showed a high sensitivity and a wide linear range of $10^{-9} \sim 10^{-5} \mathrm{M}$ and $10^{-5} \sim 10^{-2} \mathrm{M}$ with the correlation coefficients of 0.994 and 0.992 , respectively. The detection limit was $3.2 \times 10^{-10} \mathrm{M}(\mathrm{RSD} 4.12 \%)$. Due to its rapidity, sensitivity and low cost, the proposed aptasensor could be used as a pre-scanning method in TET determination for the analysis of livestock products.
\end{abstract}

Keywords: Aptasensor; Tetracycline; Colloidal gold nanoparticle; Chitosan

Citation: Guanghui Shen, Yemin Guo, Xia Sun and Xiangyou Wang, "Electrochemical Aptasensor Based on Prussian Blue-Chitosan-Glutaraldehyde for the Sensitive Determination of Tetracycline", Nano-Micro Lett. 6(2), 143-152 (2014). http://dx.doi.org/10.5101/nml.v6i2.p143-152

\section{Introduction}

Tetracyclines (TCs) have been extensively used as a bacteriostatic and antibiotic drug in livestock production to control bacterial infections and to increase the growth rate of animals owing to its broad-spectrum antibacterial activity [1]. Tetracycline (TET), oxytetracycline hydrochloride (OTC), chlortetracycline (CTC) and doxycycline hydrochloride (DXC) are members of the polyketide family, which includes a number of important pharmaceutical compounds. They are widely used as bactericidal agents in both human and veterinary medicine during recent decades [2]. However, the heavy use of TET and other TCs in aquaculture and farming industry may lead to the antibiotics residues in diary food products [3-6]. It has been concerned that the use of antibiotics in food-producing animals, particularly long-term use for growth promotion, contributes to the emergence of antibiotic-resistant bacteria in animals. These resistant bacteria may spread from animals to human beings via the food chain. They may also transfer their antibiotic-resistant genes into human pathogenic bacteria, leading to failure risk of antibiotic treatment for possibly life-threatening human conditions [7]. To safeguard human health, many organizations in the world have officially established a maximum antibiotics residues limit of $100 \mu \mathrm{g} / \mathrm{kg}$ in the foodproducing species [8-10]. So it is of grave importance

School of Agricultural and Food Engineering, Shandong University of Technology, Zibo, Shandong 255049, China

*Corresponding author: E-mail: sunxia2151@sina.com 
to develop a quick and convenient method to test and identify antibiotics residues in livestock products.

Over the past decade, many studies had been established for the determination of TET residues. Kurittu et al. [11] used microbiological inhibition test technique to measure TET residues, but the test lacked specificity and sensitivity. Therefore, a lot of traditional analytical methods have been developed for the replacement of microbiological assays, such as high performance liquid chromatography (HPLC) [12-14], capillary electrophoresis (CE) $[15,16]$, fluorescence (FL) $[17,18]$ and mass spectrometric detection (MS) [19,20]. These traditional analytical methods are highly sensitive and specific, but they are not suitable for widespread and rapid detection. Because they need expensive equipments and complicated procedures for sample pretreatment. In view of these disadvantages, a simple, sensitive and selective method for the determination of TET residue is required.

In recent years, new rapid electrochemical methods for TET detection appeared frequently, such as immunosensors [21], enzyme sensors [22] and aptasensor [23]. Aptamers are nucleic acid ligands selected from random sequence pools in vitro, which can be naturally folded into different three-dimensional structures and have the capability of binding specifically to biosurfaces [24-27]. Aptamers against a specific target can be obtained by an iterative approach called Systematic Evolution of Ligands by Exponential Enrichment (SELEX) [25]. Compared to antibodies, aptamers have more advantages, such as low cost, stability, simplicity, easy modification, target versatility and can be synthetically manufactured. To the best of our knowledge, electrochemical aptasensors for TET detection have been applied to detect TET in the analysis of the food safety [23,28]. Kim et al. [28] developed a electrochemical aptasensor based on the screen printed gold electrode using square-wave voltammetry (SWV) technique for the detection of TET, but the proposed aptasensor suffered the drawbacks of low sensitivity, and the stability and reproducibility of the method were not discussed.

Nanometer materials, as the new modified sensing interface, can facilitate the immobilization of biomolecules and improve electrochemical properties of the transducer, such as owning low-background current, high signal to noise ratio and fast electron transfer [29]. Advanced inorganic nanoparticles are currently the most popular material with a wide range of application including optics, electricity and sensors [30]. Among various nanomaterials, colloidal gold nanoparticles (AuNPs) have gained considerable attention because of their large specific surface area, good biocompatibility and high surface free energy [31,32]. Chitosan (CS) possesses excellent membrane-forming ability, high permeability towards water, good adhesion, outstanding biocompatibility, nontoxicity, high me- chanical strength and susceptibility to chemical modification due to the protonation and insolubility in solution with $\mathrm{pH}$ above $\mathrm{pKa}$ (6.3) for the deprotonation [33]. It possesses abundant primary amino groups, so it has a high affinity for Au.

In this work, we proposed a novel label-free electrochemical aptasensor based on prussian blue (PB) as the signal for the determination of TET. Herein, PBchitosan-glutaraldehyde (PB-CS-GA) was synthesized for the first layer modificatory material of the electrode, which could adsorb AuNPs and act as "molecular wire" to facilitat the electron transfer. AuNPs serving as the second layer modificatory material were used for two purposes: (a) to further accelerate the electron transfer (b) to bond and immobilize the anti-TET aptamer onto the electrode via $\mathrm{Au}-\mathrm{N}$ bond. TET was captured by the anti-TET aptamer immobilized AuNPs/PB-CSGA modified electrode, which was folded into specific three-dimensional structures. Each step modification of the electrode was investigated by cyclic voltammetry $(\mathrm{C}-\mathrm{V})$, and the concentration of TET was analyzed by differential pulse voltammetry (DPV). Due to the inhibition ability of the charge transfer of non-conducting biomolecules, the decrease level of peak currents before and after the TET captured by anti-TET aptamer indicated the concentration of TET. The constructed aptasensor preparation process and determination ability toward its target were studied in detail and it was applied to determine TET in milk samples with satisfactory results.

\section{Experimental}

\section{Materials}

The anti-TET oligonucleotides purchased from Shanghai Sangon Biological Engineering Technology \& Services Co., Ltd. (Shanghai, China) with the following sequences were designed by Niazi [34] and Zhou [23]: 5'- $\mathrm{NH}_{2}-\left(\mathrm{CH}_{2}\right)-\mathrm{CGT}$ ACG GAA TTC GCT AGC CCC CCG GCA GGC CAC GGC TTG GGT TGG TCC CAC TGC GCG TGG ATC CGA GCT CCA CGT G -3' and probe aptamer 5'-GCA TGC CTT AAG CGA TCG GGG GGC CGT CCG GTG CCG AAC CCA ACC AGG GTG ACG CGC ACC TAG GCT CGA GGT GCA C-6 FAM (FITC)-3' Chloroauric acid $\left(\mathrm{HAuCl}_{4}\right)$ and ethanol were obtained from Sinopharm Chemical Reagent Co., Ltd. (Shanghai, China). Chitosan (CS) was purchased from Sangon Biotech Co., Ltd. (Shanghai, China). Phosphate buffer solution (PBS, $\mathrm{pH}=7.4$ ) was prepared from $1.45 \mathrm{~g}$ $\mathrm{Na}_{2} \mathrm{HPO}_{4} \cdot 12 \mathrm{H}_{2} \mathrm{O}, 0.1 \mathrm{~g} \mathrm{KH}_{2} \mathrm{PO}_{4}, 0.1 \mathrm{~g} \mathrm{KCl}$ and $4 \mathrm{~g}$ $\mathrm{NaCl}$ in $500 \mathrm{~mL}$ ultrapure water with its $\mathrm{pH}$ adjusted by $\mathrm{HCl}$ and $\mathrm{NaOH}$ solutions. The other chemicals were analytical reagent grade and used without further purification. Ultrapure water $(18.2 \mathrm{M} \Omega \cdot \mathrm{cm})$ was produced 
by Millipore (PALL, Germany).

\section{Apparatus and measurements}

The $\mathrm{C}-\mathrm{V}$ and differential pulse voltammetry (DPV) were carried out on a CHI660D electrochemical workstation (Chenhua Co., Shanghai, China). A threeelectrode system was selected to measure electrochemical response. Modified glass carbon electrode (GCE) (3 $\mathrm{mm}$ in diameter) was used as working electrode. Platinum wire acted as auxiliary electrode, and saturated calomel electrode was used as reference electrode. Scanning electron microscope (SEM) was carried out on JSM-6360LV SEM (Japan). Fourier transformation infrared (FT-IR) spectroscopy was recorded with a Nicolet 380 FTIR spectrometer (Thermo Fisher Scientific Co., USA).

\section{Preparation of colloidal gold nanoparticles}

The colloidal AuNPs were prepared according to the reference with slight modifications [35]. First of all, all glassware used in these preparations needed to be thoroughly cleaned in aqua regia $\left(\mathrm{HCl}: \mathrm{HNO}_{3}=3: 1\right)$. Then the glassware was rinsed with ultrapure water, and oven-dried prior to be used. Then $2.5 \mathrm{~mL}$ of $1 \%$ sodium citrate solution was quickly added to $100 \mathrm{~mL}$ $0.01 \% \mathrm{HAuCl}_{4}$ solution, and the mixture solution was brought to boiling with vigorous stirring. Then the color changed from pale-yellow to claret-red within 2 min. Keeping the solution boiling for $10 \mathrm{~min}$, the mixture was continued to be stirred for another $10 \mathrm{~min}$ until the solution returning to room temperature. Finally, the colloidal AuNPs were stored in a refrigerator with a dark-colored glass bottle.

\section{Preparation of CS-GA composites}

$1 \%(1 \mathrm{~g} / 100 \mathrm{~mL})$ chitosan solution was prepared by dissolving $1.0 \mathrm{~g}$ chitosan flakes into $100 \mathrm{~mL} 1.0 \%$ acetic acid and stirring for $3 \mathrm{~h}$. Then 5\% GA solution was added into the $1 \% \mathrm{CS}$ solution by 10 min sonication to get CS-GA solution. The solution was stored in refrigerator.

\section{Fabrication of the aptasensor}

The GCE was sonicated in "piranha solution" $\left(\mathrm{H}_{2} \mathrm{SO}_{4}: 30 \% \mathrm{H}_{2} \mathrm{O}_{2}=7: 3, \mathrm{~V} / \mathrm{V}\right)$, and rinsed with ultrapure water before dried naturally. Next, the GCE was polished to a mirror-like appearance with $0.05 \mu \mathrm{m}$ alumina slurry, and then sonicated in nitric acid $(\mathrm{V} / \mathrm{V}$, 1:1), ethanol and ultrapure water for $5 \mathrm{~min}$, respectively. Firstly, the bare GCE was immerged in the CS-GA solution containing $2.5 \mathrm{mM} \mathrm{FeCl}_{3}, 2.5 \mathrm{mM}$ $\mathrm{K}_{3}\left[\mathrm{Fe}(\mathrm{CN})_{6}\right], 0.1 \mathrm{M} \mathrm{KCl}, 0.01 \mathrm{M} \mathrm{HCl}$ with a constant potential of $0.4 \mathrm{~V}$ for electrodeposition for $300 \mathrm{~s}$. PB was deposited onto the surface of the electrode when $\mathrm{FeCl}_{3}\left(\mathrm{Fe}^{3+}\right)$ and $\mathrm{K}_{3}\left[\mathrm{Fe}(\mathrm{CN})_{6}\right]\left(\mathrm{Fe}(\mathrm{CN})_{6}^{3-}\right)$ were coexis- tent in the electrodeposition solution, and the reactions were as follows:

$$
\mathrm{Fe}^{3+} \stackrel{+\mathrm{e}}{\longrightarrow} \mathrm{Fe}^{2+} \stackrel{\mathrm{Fe}(\mathrm{CN})_{6}^{3-}}{\longrightarrow} \mathrm{KFe}\left[\mathrm{Fe}(\mathrm{CN})_{6}\right]
$$

After that, the electrode was cyclic scanned in the potential range from -0.2 to $+0.6 \mathrm{~V}$ at the scan rate of $50 \mathrm{mV} / \mathrm{s}$ until the $\mathrm{C}-\mathrm{V}$ curve stable, and the PBCS-GA/GCE was obtained. Then $5 \mu \mathrm{L}$ AuNPs were added onto the surface of PB-CS-GA/GCE and dried in the air at room temperature (noted as AuNPs/PBCS-GA/GCE). Then the AuNPs/PB-CS-GA/GCE removed physically adsorbed colloidal AuNPs with ultrapure water and dried by nitrogen. $5 \mu \mathrm{L}$ anti-TET aptamer of $5 \mathrm{mM}$ was dropped onto the AuNPs/PB-CSGA/GCE. Then a rubber cap was coated on the electrode to prevent evaporation of the aptamer solution. $4 \mathrm{~h}$ later, unbound anti-TET aptamer was removed by ultrapure water and then anti-TET/AuNPs/PB-CSGA/GCE was passivated by $1 \mathrm{M}$ ethanolamine (EA) for $1 \mathrm{~h}$ to obtain EA/anti-TET/AuNPs/PB-CS-GA/GCE (ethanolamine was used to remove non-specific adsorption on the anti-TET/AuNPs/PB-CS-GA/GCE surface). Finally, the EA/anti-TET/AuNPs/PB-CS$\mathrm{GA} / \mathrm{GCE}$ was rinsed with ultrapure water and stored at $4^{\circ} \mathrm{C}$ in refrigerator. The obtained electrode was used as the aptasensor in this work, and the stepwise assembly of the proposed aptasensor was shown in Scheme 1 .

\section{Hybridization of anti-TET aptamer with probe aptamer}

$5 \mu \mathrm{L}$ anti-TET aptamer with $5 \mathrm{mM}$ concentration was dropped onto the AuNPs/PB-CS-GA modified glass slide. $4 \mathrm{~h}$ latter, $2 \mu \mathrm{L}$ probe aptamer $(2 \mathrm{mM})$ was added and the hybridization reaction was carried out at room temperature in the dark environment for $2 \mathrm{~h}$. The obtained sample was rinsed with $0.1 \mathrm{M}$ Tris$\mathrm{HCl}$ buffer to remove unbound probe aptamer, and then dried in the air atmosphere.

\section{Electrochemical measurements}

The electrochemical characteristics of the modified electrode were characterized by $\mathrm{C}-\mathrm{V}$ and DPV in PBS $(\mathrm{pH}=7.4)$. Electrochemical measurements were established in a conventional electrochemical cell. The formation of anti-TET/TET complexes was performed by dropped $10 \mu \mathrm{L}$ of different concentration of the target TET on the EA/anti-TET/AuNPs/PB-CS-GA/GCE at $37^{\circ} \mathrm{C}$ for $1 \mathrm{~h}$. After being carefully washed with ultrapure water to remove unbound TET, the electrode was characterized by DPV for the detection of TET. The DPV measurements were performed in the potential range from $-0.1 \mathrm{~V}$ to $+0.4 \mathrm{~V}$ with pulse amplitude of $50 \mathrm{mV}$. 


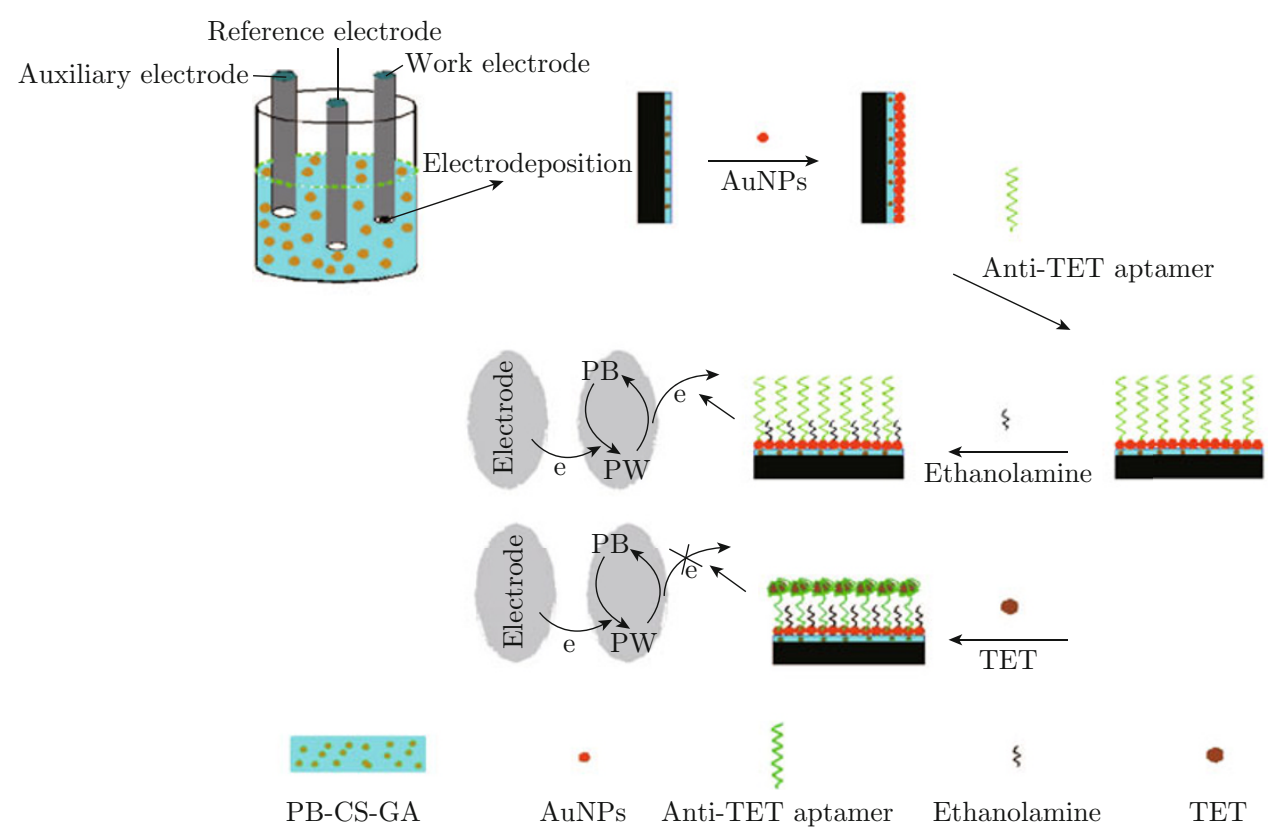

Scheme 1 The stepwise fabrication process of the aptasensor.

\section{Results and discussion}

\section{Sensing mechanisms of the aptasensor}

In this study, the aptasensor was constructed by covalently attaching an amino-modified anti-TET aptamer on the AuNPs/PB-CS-GA coated GCE surface. It contained 76-base sequence and specifically binded to TET with high affinity. The AuNPs were employed as the carriers of the anti-TET aptamer to amplify the change of peak currents. The PB was used as a mediator to generate the electron flow between bulk solution and work electrode, which was shown in Scheme 1. The anti-TET aptamer modified electrodes presented a stable and free configuration on the GCE surface to give a significantly strong faradaic current. The formation of anti-TET/TET complex hindered the electron-transfer for the PB and Prussian white (PW) transition, thus producing a detectable decreasing signal. The electrochemical data analysis was carried out by the change of the DPV peak currents before and after the TET treatment in PBS $(\mathrm{pH}=7.4)$. The reaction between $\mathrm{PB}$ and $\mathrm{PW}$ was as follows:

$$
\begin{gathered}
\mathrm{KFe}^{\mathrm{III}}\left[\mathrm{Fe}^{\mathrm{II}}(\mathrm{CN})_{6}\right]+\mathrm{e}^{-}+\mathrm{K}^{+} \leftrightarrow \mathrm{K}_{2} \mathrm{Fe}^{\mathrm{II}}\left[\mathrm{Fe}^{\mathrm{II}}(\mathrm{CN})_{6}\right] \\
\mathrm{PB}
\end{gathered}
$$

\section{Morphology characterization of the aptasensor}

The surface morphology of the PB-CS-GA/GCE and AuNPs/PB-CS-GA/GCE were characterized by SEM. The SEM image of the PB-CS-GA modified GCE was shown in Fig. 1(a). It exhibits the morphology with a rough, porous, and homogeneous layer with packed uniform particles. When the AuNPs were covered over the PB-CS-GA modified GCE, the surface exhibited a film with many globular features (Fig. 1(b)). Furthermore, to monitor anti-TET aptamer immobilizing process, fluorescent images before and after the probe aptamer assembly were indicated. In Fig. 1(c) and Fig. 1(d), the results showed that the FITC labeled anti-TET aptamer immobilized on AuNPs/PB-CS-GA modified glass released visible green fluorescence under the fluorescent microscopy, while the anti-TET aptamer covered AuNPs/PB-CS-GA modified glass exhibited no fluorescence.

\section{Characterization of the aptasensor}

The FT-IR was applied to character the formation and properties of the films. FT-IR spectra of CS, PB and PB-CS-GA were measured and shown in Fig. 2. It could be seen in FT-IR spectra of CS (Fig. 2(a)), the band around the wave number of $3400 \mathrm{~cm}^{-1}$ showed an obvious absorption peak which could be attributed to the stretching vibration of $-\mathrm{OH}$ or $-\mathrm{NH}$ groups. The absorption peaks around the wave number of $1560 \mathrm{~cm}^{-1}$ and $1400 \mathrm{~cm}^{-1}$ corresponded to the bending vibration of $\mathrm{N}-\mathrm{H}$ and $\mathrm{C}-\mathrm{H}$, respectively. The FT-IR spectra in Fig. 2(b) showed stretching vibration of - CN group which was contained in molecule of PB from 2080 to $2040 \mathrm{~cm}^{-1}$. Figure 2(c) contained all the characteristic peaks appeared in CS and PB, and another typical absorbance band attributed to stretching vibration of $-\mathrm{C}=\mathrm{O}$ group (around $1640 \mathrm{~cm}^{-1}$ ) existing in GA, which indicated that the PB-CS-GA was successfully deposited onto the surface of the electrode. 

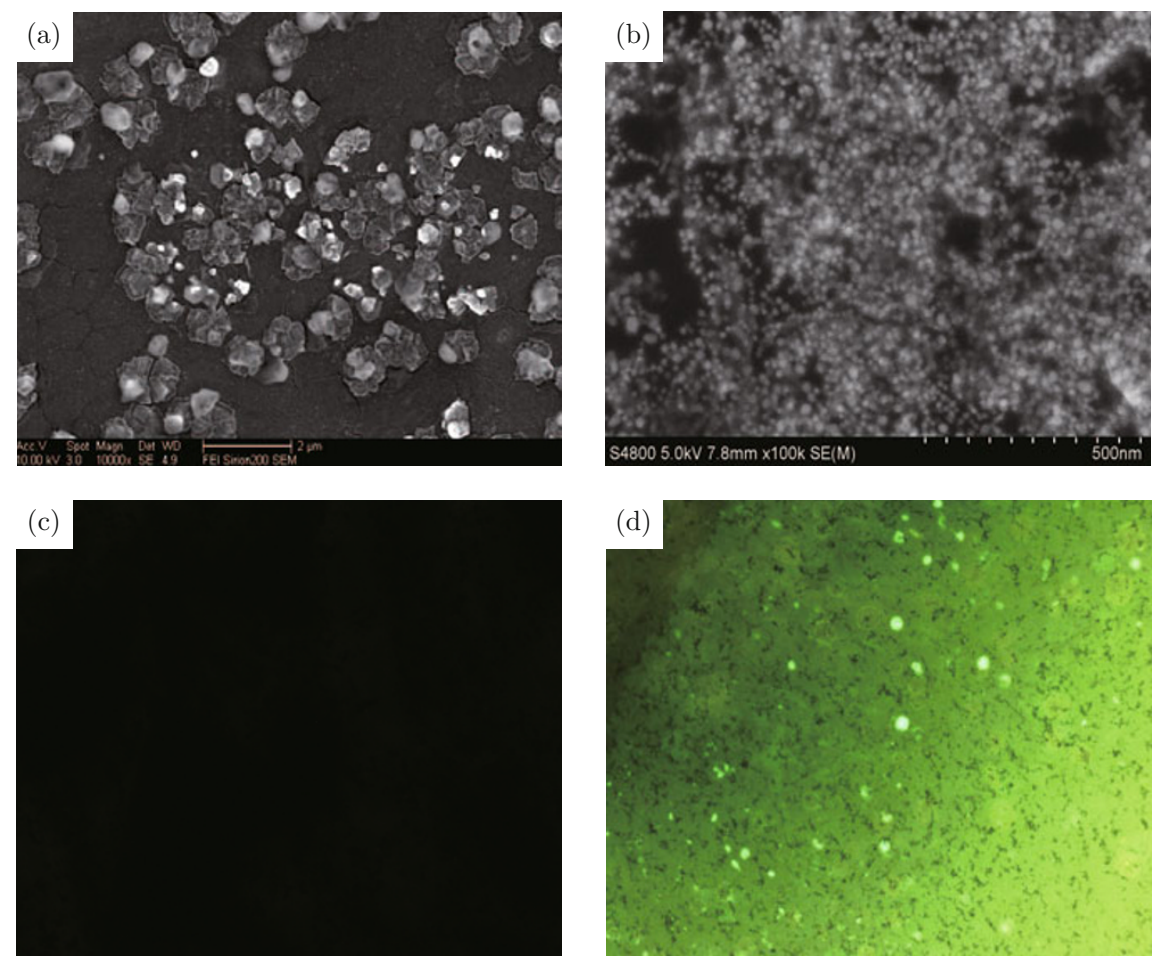

Fig. 1 SEM images of (a) PB-CS-GA/GCE; (b) AuNPs/PB-CS-GA/GCE; Fluorescence microscope images of the anti-TET modified glass (c) and the probe aptamer hybridized with the anti-TET (d).

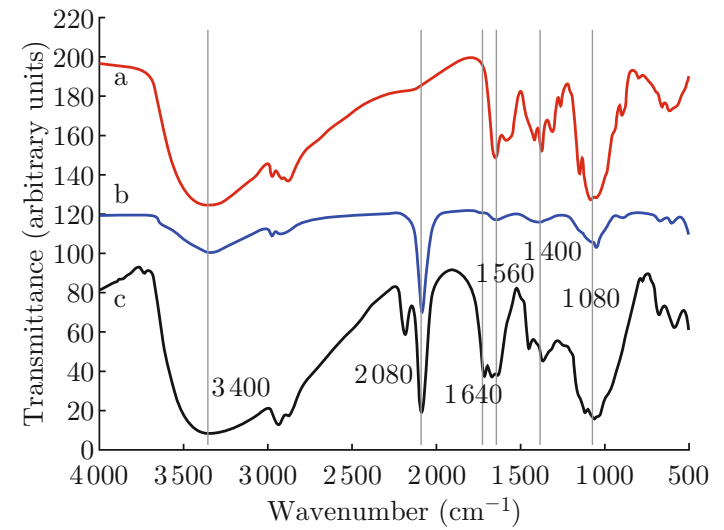

Fig. 2 The FT-IR spectra of (a) CS; (b) PB and (c) PBCS-GA.

$\mathrm{C}-\mathrm{V}$ is an effective and convenient technique for probing the feature of the modified electrode surface. Herein $\mathrm{C}-\mathrm{V}$ was employed to investigate the electrochemical properties of each immobilization step. Figure 3 showed the $\mathrm{C}-\mathrm{Vs}$ of different electrodes in the PBS buffer $(\mathrm{pH}=$ 7.4). No redox peak can be observed for the bare GCE (Fig. 3(a)). Figure 3(b) showed reversible oxidationreduction peaks of $\mathrm{PB}$ in $\mathrm{PBS}$. Compared to the bare $\mathrm{GCE}$, the electrochemical response of the electrode indicated the conversion of $\mathrm{PW}$ and $\mathrm{PB}$ on the electrode. The current clearly increased when AuNPs were deposited on the electrode, indicating that the use of AuNPs significantly enhanced the conductivity of the electrode as a conductive wire or an electron-conducting tunnel (Fig. 3(c)). While the anti-TET aptamer was immobilized successfully on the modified electrode, the peak currents decreased (Fig. 3(d)). This was because the immobilized anti-TET aptamer hindered electron transmission. After EA was used to block nonspecific sites and the TET was captured by the anti-TET aptamer, the diffusion of the conductive ion participated in the reaction between $\mathrm{PB}$ and $\mathrm{PW}$ was hindered, so the peak current further decreased in turn (Fig. 3(e) and $3(\mathrm{f}))$.

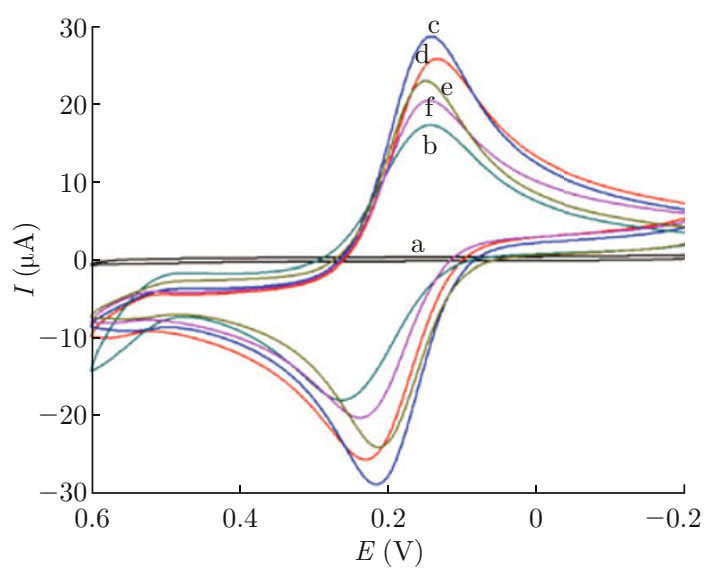

Fig. $3 \mathrm{C}-\mathrm{Vs}$ of the bare GCE (a); PB-CS-GA/GCE (b); AuNPs/PB-CS-GA/GCE (c); anti-TET/AuNPs/PBCS-GA/GCE (d); EA/anti-TET/AuNPs/PB-CS-GA/GCE (e) and TET/EA/anti-TET/AuNPs/PB-CS-GA/GCE (f) in the $\mathrm{pH}$ 7.4 $\mathrm{PBS}$ in the potential range of potential range from -0.2 to $+0.6 \mathrm{~V}$ at scan rate of $50 \mathrm{mV} / \mathrm{s}$. 


\section{Optimization of experimental conditions for ap- tasensor}

\section{Optimal concentration of the anti-TET for the aptasensor}

The concentration of the anti-TET was optimized to obtain a high sensitivity of the fabricated electrochemical aptasensor. The effect of the concentration of antiTET on $\Delta I$ (the decrease of DPV peak currents before and after the TET treatment.) was investigated from $2 \mu \mathrm{M}$ to $6 \mu \mathrm{M}$. The results were shown in Fig. 4(a). It could be seen that the $\Delta \mathrm{I}$ of aptasensor increased rapidly with the increase of anti-TET concentration in the range of $2 \sim 5 \mu \mathrm{M}$. After the concentration was higher than $5 \mu \mathrm{M}$, the $\Delta I$ almost kept constant, which indicated $5 \mu \mathrm{M}$ anti-TET was sufficient to cover the AuNPs/PB-CS-GA modified GCE surface. Therefore, $5 \mu \mathrm{M}$ anti-TET was selected as the optimum loading amount in this study.
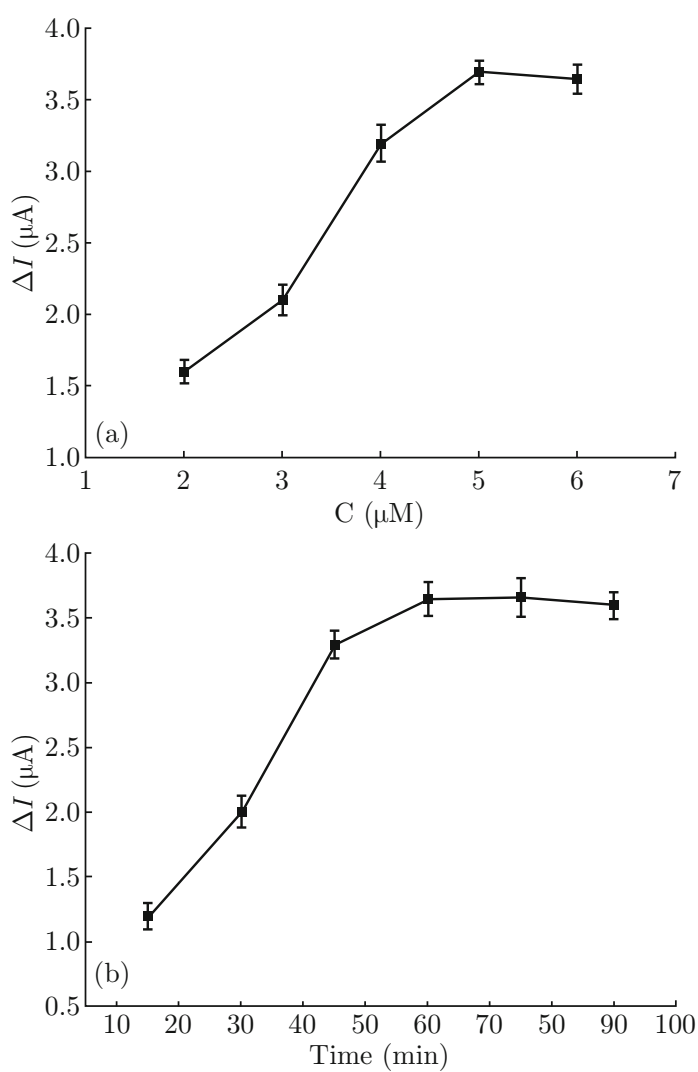

Fig. 4 Optimization of the experimental parameters: effects of (a) the concentration of the anti-TET and (b) the TET incubation time.

\section{Optimal incubation time for the aptasensor}

The incubation time of the anti-TET with the TET was an important parameter for the aptasensor. It could be seen that a visible difference in the increase of $\Delta I$ with different incubation times. As shown in
Fig. 4(b), the $\Delta I$ increased gradually with the augment of incubation time. However, when the incubation time reached $60 \mathrm{~min}$, the signal response sustained a stable value. The result indicated that the interaction of aptamer with TET reached to equilibrium. Thus, the incubation time was controlled to be $60 \mathrm{~min}$.

\section{Performance of the aptasensor}

\section{Detection of TET}

DPV technique could provide a better peak resolution and current sensitivity, which was very suitable for detecting the concentration of TET. The performed aptasensors were evaluated by the relationship between the $\Delta I$ and TET concentrations. Under the optimal experimental conditions, DPVs for TET detection were obtained. As shown in Fig. 5(a), the decrease of peak current was proportional to the logarithm of the TET
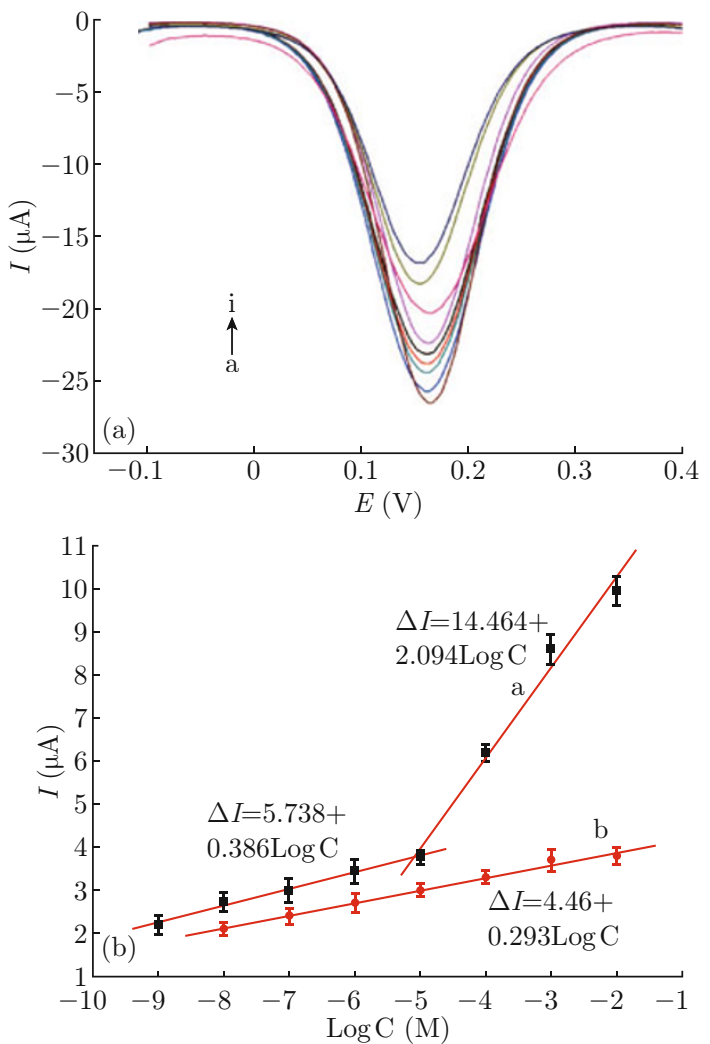

Fig. 5 (a) DPVs of the aptasensor incubated with different concentrations of TET (a-i: the concentrations of TET are $0 \mathrm{M}, 1 \times 10^{-9} \mathrm{M}, 1 \times 10^{-8} \mathrm{M}, 1 \times 10^{-7} \mathrm{M}, 1 \times 10^{-6} \mathrm{M}$, $1 \times 10^{-5} \mathrm{M}, 1 \times 10^{-4} \mathrm{M}, 1 \times 10^{-3} \mathrm{M}, 1 \times 10^{-2} \mathrm{M}$ ) were performed in the $\mathrm{pH} 7.4 \mathrm{PBS}$ in the potential range from $-0.1 \mathrm{~V}$ to $+0.4 \mathrm{~V}$ with the parameters: pulse amplitude 50 $\mathrm{mV}$, pulse width $0.2 \mathrm{~s}$, pulse period $0.5 \mathrm{~s}$; (b) The linear relationship between the peak current changes $(\Delta I)$ in the DPV and the logarithmic values of the TET concentrations. Error bars showed the standard deviations of measurements taken from three independent experiments. 
Nano-Micro Lett. 6(2), 143-152 (2014)/http://dx.doi.org/10.5101/nml.v6i2.p143-152

Table 1 Comparison with other methods for the determination of TET

\begin{tabular}{lccc}
\hline Method of detection & Limit of detection $(\mathrm{M})$ & Linear range $(\mathrm{M})$ & References \\
\hline Fluorescence optical fiber sensor & $1.06 \times 10^{-7}$ & $6.98 \times 10^{-7}-8.73 \times 10^{-5}$ & {$[37]$} \\
Molecularly Imprinted Technique & $5.58 \times 10^{-8}$ & $2.25 \times 10^{-7}-2.25 \times 10^{-5}$ & {$[38]$} \\
high-throughput suspension array technology & $3.4 \times 10^{-9}$ & $1.5 \times 10^{-8}-6.7 \times 10^{-6}$ & {$[39]$} \\
high pressure liquid chromatographic & $2.1 \times 10^{-8}$ & $1 \times 10^{-9}-1 \times 10^{-2}$ & this work \\
aptasensor & $3.2 \times 10^{-10}$ & & the \\
\hline
\end{tabular}

concentration. In Fig. 5(b), curve aindicates the current variables of DPV showing a good linear relationship with the logarithmic values of the TET concentrations in two ranges of $1 \times 10^{-9} \mathrm{M} \sim 1 \times 10^{-5} \mathrm{M}$ and $1 \times 10^{-5} \mathrm{M} \sim 1 \times 10^{-2} \mathrm{M}$. The linear equation was $\Delta I(\mu \mathrm{A})=5.738+0.386 \log C(\mathrm{M})$ and $\Delta I(\mu \mathrm{A})=$ $14.464+2.094 \log C(\mathrm{M})$, with a correlation coefficient of 0.994 and 0.992 , respectively. The limit of detection (LOD) of TET was calculated as three times higher than the standard deviation for the average measurements of blank samples (LOD $=3 \mathrm{RSD} /$ slope) [36]. Therefore, the LOD was determined to be as low as $3.2 \times 10^{-10} \mathrm{M}$ with relative standard deviation (RSD) of $4.12 \%$, lower than that of TET detection in other ways showed in Table 1. As comparison, the current responses of the aptasensor (without PB-CS-GA composite, EA/anti-TET/AuNPs/GCE, curve b in Fig. 5(b)) were recorded in $0.1 \mathrm{~mol} / \mathrm{L} \mathrm{PBS}(\mathrm{pH}=7.4)$ containing $5.0 \mathrm{mM} \mathrm{K}_{3}\left[\mathrm{Fe}(\mathrm{CN})_{6}\right]$, and the linear range was $1 \times 10^{-8}$ $\mathrm{M} \sim 1 \times 10^{-2} \mathrm{M}$ with the detection limit of $7.4 \times 10^{-9}$ M. It was observed that the EA/anti-TET/AuNPs/PBCS-GA modified electrode leaded a wider dynamic measurement range and a higher sensitivity compared with EA/anti-TET/AuNPs modified electrode. Based on the above work, we could conclude that $\mathrm{PB}$ mixed into CS-GA as the signal indicator could improve the sensitivity of the electrochemical aptasensor.

\section{The specificity of the aptasensor}

The specificity performance of the aptasensor for TET was further studied in this work. The selectivity test was carried out by measuring and comparing the response of TET $(5 \mu \mathrm{M})$ to some possible interferences including a structurally similar tetracycline derivative OTC $(5 \mu \mathrm{M})$ and two structurally distinct molecules of Kanamycin Monosulfate $(5 \mu \mathrm{M})$ and Gentamycin Sulfate $(5 \mu \mathrm{M})$. It could be observed in Fig. 6 , the TET sample existed a significant peak current change, while the same concentrations of the other three chemicals had slight emissions. These tests indicated that the developed strategy could be used to identify TET with high specificity.

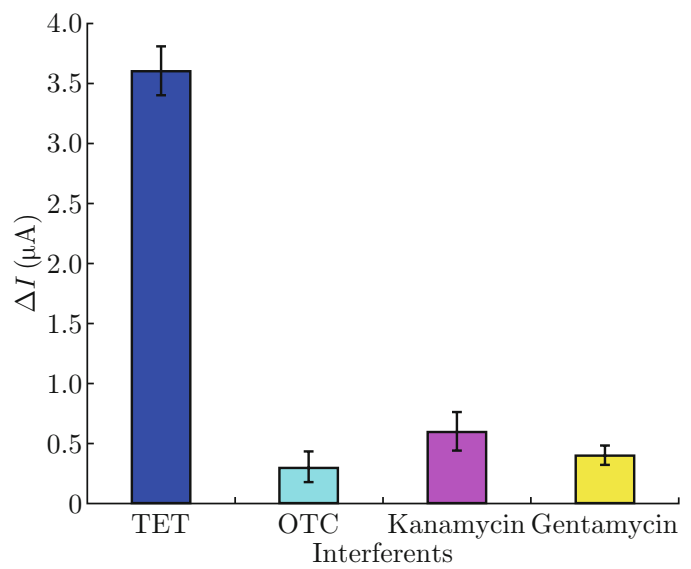

Fig. 6 The specificity of the aptasensor in the presence of $5 \mu \mathrm{M}$ TET, $5 \mu \mathrm{M}$ OTC, $5 \mu \mathrm{M}$ Kanamycin Monosulfate, 5 $\mu \mathrm{M}$ Gentamycin Sulfate.

\section{Stability and reproducibility of the aptasensor}

The stability of the aptasensor was studied by comparing the $\Delta I$ after a storage period of 7,14 and 21 days. The electrodes were stored in refrigerator at $4^{\circ} \mathrm{C}$ and $\Delta I$ was periodically measured. The response current retained above $96 \%, 93 \%$ and $89 \%$ of its initial response, respectively, which indicated the storage stability of the aptasensor was quite good.

The reproducibility of the aptasensor was evaluated by detecting the $\Delta I$ response with five electrodes prepared in the same way. The five aptasensors were incubated with $10^{-5} \mathrm{M}$ TET, and an acceptable reproducibility with relative standard deviation (RSD) of $5.8 \%$ was obtained. The experimental result indicated the proposed aptasensor had a good reproducibility.

\section{Determination of TET in milk samples}

In order to evaluate the feasibility of the aptasensor system for possible applications, the standard addition method was used. The milk samples were prepared as follows: the milk was firstly diluted at the ratio of 1:10 and ultracentrifugated at $30000 \mathrm{rpm}$ for $90 \mathrm{~min}$. In this way, the intermediate layer milk serum was obtained without fat and casein. Then we added TET to the collected TET-free milk serum to the final concen- 
tration of $5 \times 10^{-8} \mathrm{M}, 5 \times 10^{-6} \mathrm{M}$ and $5 \times 10^{-4} \mathrm{M}$. As shown in Table 2, the recovery tests were performed using 3 replicates with the aptasensor that prepared in the same way. The recovery of $92 \sim 106 \%$ indicated that the aptasensor was available for the analysis of TET in milk samples.

Table 2 Determination of TET in milk samples

\begin{tabular}{ccccc}
\hline Sample & $\begin{array}{c}\text { Added } \\
(\mathrm{M})\end{array}$ & $\begin{array}{c}\text { Total } \\
\text { found }(\mathrm{M})\end{array}$ & $\begin{array}{c}\text { RSD } \\
(\%, \mathrm{n}=3)\end{array}$ & $\begin{array}{c}\text { Recovery } \\
(\%)\end{array}$ \\
\hline 1 & $5 \times 10^{-8}$ & $4.8 \times 10^{-8}$ & 5.3 & 96 \\
2 & $5 \times 10^{-6}$ & $5.3 \times 10^{-6}$ & 6.2 & 106 \\
3 & $5 \times 10^{-4}$ & $4.6 \times 10^{-4}$ & 5.4 & 92 \\
\hline
\end{tabular}

\section{Conclusions}

In this work, a novel label-free aptasensor based on AuNPs/PB-CS-GA for sensitive determination of tetracycline was developed. The PB-CS-GA has performed higher sensitivity of the electrochemical aptasensor due to the PB as the signal indicator. In addition, the PBCS-GA composites were stable and could be easily prepared. Compared to the electrochemical aptasensor for TET reported by Kim et al. [28]. The AuNPs/PBCS-GA/GCE system showed good conductibility and biocompatibility and exhibited higher sensitivity and stability. Its electrochemical signal had a good linear relationship with the TET concentration in the range of $1 \times 10^{-9} \mathrm{M} \sim 1 \times 10^{-5} \mathrm{M}$ and $1 \times 10^{-5} \mathrm{M} \sim 1 \times 10^{-2}$ M. This strategy contributed to improve the sensitivity and stability of aptasensor, thus, it provided a novel promising platform of aptasensor for TET detection.

\section{Acknowledgements}

This work was supported by the National Natural Science Foundation of China (No. 30972055, 31101286), and Agricultural Science and Technology Achievements Transformation Fund Projects of the Ministry of Science and Technology of China (No. 2011GB2C60020).

\section{References}

[1] K. Kishida, "Simplified extraction of tetracycline antibiotics from milk using a centrifugal ultrafiltration device", Food Chem. 126(2), 687-690 (2011). http:// dx.doi.org/10.1016/j.foodchem. 2010.11.021

[2] D. E. Brodersen, W. M. Jr. Clemons, A. P. Carter, R. J. Morgan-Warren, B. T. Wimberly and V. Ramakrishnan, "The structural basis for the action of the antibiotics tetracycline, pactamycin, and hygromycin B on the $30 \mathrm{~S}$ ribosomal subunit", Cell 103(7), 1143-1154 (2000). http://dx.doi.org/10. 1016/S0092-8674(00) 00216-6
[3] F. K. Muriuki, "Tetracycline residue levels in cattle meat from Nairobi salughter house in Kenya", J. Vet. Sci. 2(2), 97-101 (2001).

[4] N. Vragovic, D. Bazulic and B. Njari, "Risk assessment of streptomycin and tetracycline residues in meat and milk on Croatian market", Food Chem. 49(2), 352-355 (2011). http://dx.doi.org/10.1016/j.fct. 2010.11 .006

[5] M. Kuhne, S. Wegmann, A. Kobe and R. Fries, "Tetracycline residues in bones of slaughtered animals", Food Control. 11(3). 175-180 (2000). http://dx.doi.org/ 10.1016/S0956-7135(99)00092-4

[6] G. T. Peres, S. Rath and F. G. Reyes, "A HPLC with fluorescence detection method for the determination of tetracyclines residues and evaluation of their stability in honey", Food Control. 21(5), 620-625 (2010). http://dx.doi.org/10.1016/ j.foodcont. 2009.09.006

[7] J. G. Salisbury, T. J. Nicholls, A. M. Lammerding, J. Turnidge, M. J. Nunn, "A risk analysis framework for the long-term management of antibiotic resistance in food-producing animals". Int. J. Antimicrob. Agents 20(3), 153-164 (2002). http://dx.doi.org/10.1016/ S0924-8579 (02) 00169-3

[8] Commission Regulation 508/1999/EC, 1999.

[9] Japanese Ministry of Health Welfare and Labor, 2008.

[10] US Food and Drug Administration, 1975.

[11] J. Kurittu, S. Lönnberg, M. Virta and M. Karp, "A group-specific microbiological test for the detection of tetracycline residues in raw milk". J. Agric. Food. Chem. 48(8), 3372-3377 (2000). http://dx.doi.org/ 10.1021/jf9911794

[12] J. W. Fritz and Y. Zuo, "Simultaneous determination of tetracycline, oxytetracycline, and 4-epitetracycline in milk by high-performance liquid chromatography", Food Chem. 105(3), 1297-1301 (2007). http://dx. doi.org/10.1016/j.foodchem. 2007.03.047

[13] K. Ng and S. W. Linder, "HPLC separation of tetracycline analogues: comparison study of laser-based polarimetric detection with UV detection", J. Chromatogr Sci. 41(9), 460-466 (2003). http://dx.doi. org/10.1093/chromsci/41.9.460

[14] A. C. Martel, S. Zeggane, P. Drajnudel, J. P. Faucon and M. Aubert, "Tetracycline residues in honey after hive treatment", Food Addit. Contam. 23(3), 265-273 (2006). http://dx.doi.org/10.1080/ 02652030500469048

[15] P. Kowalski, "Capillary electrophoretic method for the simultaneous determination of tetracycline residues in fish samples", J. Pharm. Biomed. 47(3), 487-493 (2008). http://dx.doi.org/10.1016/ j.jpba. 2008.01.036

[16] B. Y. Deng, Q. X. Xu, H. Lu, L. Ye and Y. Z. Wang, "Pharmacokinetics and residues of tetracycline in crucian carp muscle using capillary electrophoresis on-line coupled with electrochemiluminescence detection", Food Chem. 134(4), 2350-2354 (2012). http:// dx.doi.org/10.1016/j.foodchem. 2012.03.117 
[17] L. M. Shen, M. L. Chen and X. W. Chen, "A novel flow-through fluorescence optosensor for the sensitive determination of tetracycline", Talanta 85(3), 1285-1290 (2011). http://dx.doi.org/ 10.1016/j.talanta.2011.06.006

[18] N. Rodríguez, B. D. Real, M. Cruz Ortiz, L. A. Sarabia, A. Herrero, "Usefulness of parallel factor analysis to handle the matrix effect in the fluorescence determination of tetracycline in whey milk", Anal. Chim. Acta 632(1), 42-51 (2009). http://dx.doi.org/10.1016/j. aca. $2008 \cdot 10.051$

[19] H. Oka, Y. Ito, Y. Ikai, T. Kagami and K. Harada, "Mass spectrometric analysis of tetracycline antibiotics in foods", J. Chromatogr. A. 812(12), 309-319 (1998). http://dx.doi.org/10.1016/ S0021-9673 (97) 01278-8

[20] M. E. Dasenaki and N. S. Thomaidis, "Multi-residue determination of seventeen sulfonamides and five tetracyclines in fish tissue using a multi-stage LC-ESIMS/MS approach based on advanced mass spectrometric techniques", Anal. Chim. Acta 672(1-2), 93-102 (2010). http://dx.doi.org/10.1016/j.aca. 2010.04 .034

[21] F. Conzuelo, M. Gamella, S. Campuzano, A. Julio Reviejo and J. M. Pingarrón, "Disposable amperometric magneto-immunosensor for direct detection of tetracyclines antibiotics residues in milk", Anal. Chim. Acta 737(6), 29-36 (2012). http://dx.doi.org/10.1016/j . aca. 2012.05.051

[22] M. Jeon, J. Kim, K. J. Paeng, S. W. Park and I. R. Paeng, "Biotin-avidin mediated competitive enzymelinked immunosorbent assay to detect residues of tetracyclines in milk", Microchem. J. 88(1), 26-31 (2008). http://dx.doi.org/10.1016/j.microc.2007.09.001

[23] L. Zhou, D. J. Li, L. Gai, J. P. Wang and Y. B. Li, "Electrochemical aptasensor for the detection of tetracycline with multi-walled carbon nanotubes amplification", Sens. Actuators, B: Chem. 162(1), 201-208 (2012). http://dx.doi.org/10.1016/j.snb. 2011.12.067

[24] A. D. Ellington and J. W. Szostak, "In vitro selection of RNA molecules that bind specific ligands", Nature 346, 818-822 (1990). http://dx.doi.org/10. 1038/346818a0

[25] C. Tuerk and L. Gold, "Systematic evolution of ligands by exponential enrichment: RNA ligands to bacteriophage T4 DNA polymerase", Science 249(4968), 505510 (1990). http://dx.doi.org/10.1126/science. 2200121

[26] C. Pestourie, B. Tavitian and F. Duconge, "Aptamers against extracellular targets for in vivo applications", Biochimie 87(9-10), 921-930 (2005). http://dx.doi. org/10.1016/j.biochi. 2005.04.013

[27] C. A. Savran, S. M. Knudsen, A. D. Ellington and S. R. Manalis, "Micromechanical detection of proteins using aptamer-based receptor molecules". Anal. Chem. 76(11), 3194-3198 (2004). http://dx.doi.org/ 10.1021/ac049859f
[28] Y. J. Kim, Y. S. Kim, J. H. Niazi and M. B. Gu, "Electrochemical aptasensor for tetracycline detection", Bioprocess. Biosyst. Eng. 33(1), 31-37 (2010). http://dx.doi.org/10.1007/s00449-009-0371-4

[29] K. Kerman, M. Saito, S. Yamamura, Y. Takamura and E. Tamiya, "Nanomaterial-based electrochemical biosensors for medical applications", Trends Anal. Chem. 27(7), 585-592 (2008). http://dx.doi.org/10. 1016/j.trac. 2008.05.004

[30] Y. T. Shi, R. Yuan, Y. Q. Chai, M. Y. Tang and $\mathrm{X}$. L. He, "Amplification of antigen-antibody interactions via back-filling of HRP on the layer-bylayer self-assembling of thionine and gold nanoparticles films on Titania nanoparticles/gold nanoparticlescoated Au electrode", J. Electroanal. Chem. 604(1), 916 (2007). http://dx.doi.org/10.1016/j.jelechem. 2007.02 .027

[31] S. Y. Xu and X. Z. Han, "A novel method to construct a third-generation biosensor:self-assembling gold nanoparticles on thiol-functionalized poly(styrene-co -acrylic acid) nanospheres", Biosens. Bioelectron. 19(9), 1117-1120 (2004). http://dx.doi.org/10. 1016/j.bios. 2003.09.007

[32] S. Q. Liu, D. Leech and H. X. Ju, "Application of colloidal gold in protein immobilization, electron transfer, and biosensing", Anal. Lett. 36(1), 1-19 (2003). http://dx.doi.org/10.1081/al-120017740

[33] P. Sorlier, A. Denuzière, C. Viton and A. Domard, "Relation between the degree of acetylation and the electrostatic properties of chitin and chitosan", Biomacromolecules 2(3), 765-772 (2001). http://dx. doi.org/10.1021/bm015531+

[34] J. H. Niazi, S. J. Lee and M. B. Gu, "Single-stranded, DNA aptamers specific for antibiotics tetracyclines", Bioorg. Med. Chem. 16(15), 7245-7253 (2008). http://dx.doi.org/10.1016/j.bmc.2008.06.033

[35] K.C. Grabar, R.G. Freeman, M.B. Hommer and M.J. Natan, "Preparation and characterization of Au colloid monolayers", Anal. Chem. 67(4), 735-743 (1995). http://dx.doi.org/10.1021/ac00100a008

[36] C. Cao, J. P. Kim, B. W. Kim, H. Chae, H. C. Yoon, S. S. Yang and S. J. Sim, "A strategy for sensitivity and specificity enhancements in prostate specific antigen- $\alpha_{1}$-antichymotrypsin detection based on surface plasmon resonance", Biosens. Bioelectron. 21(11), 2106-2113 (2006). http://dx.doi.org/ $10.1016 / j$. bios. 2005.10.014

[37] Y. Wang, W. H. Liu, K. M. Wang, G. L. Shen and R. $\mathrm{Q} . Y u$. "Fluorescence optical fiber sensor for tetracycline", Talanta. 47(1), 33-42 (1998). http://dx.doi. org/10.1016/S0039-9140(98)00049-6

[38] H. Zhao, H. T. Wang, X. Quan and F. Tan, "Amperometric Sensor for Tetracycline Determination Based on Molecularly Imprinted Technique", Procedia Environmental Sciences 18, 249-257 (2013). http://dx.doi. org/10.1016/j.proenv. 2013.04.032

[39] P. Su, N. Liu, M. X. Zhu, B. A. Ning, M. Liu, Z. H. Yang, X. J. Pan and Z. X. Gao, "Simultaneous detection of five antibiotics in milk by 
high-throughput suspension array technology". Talanta 85(2), 1160-1165 (2011). http://dx.doi.org/ $10.1016 / j$.talanta. 2011.05 .040

[40] E. Karageorgou, M. Armeni, I. Moschou, and V. Samanidou, "Ultrasound-assisted dispersive ex- traction for the high pressure liquid chromatographic determination of tetracyclines residues in milk with diode array detection". Food Chem. 150(1), 328-334(2014). http://dx.doi.org/10.1016/ j.foodchem. 2013.11.008 